Schnarcher stört die Nachtruhe

\section{Aber ohne ihn schläft die Partnerin nicht besser}

Blumen $M$ et al. Effect of sleeping alone on sleep quality in female bed partners

of snorers. Eur Respir J. 2009;34:1127-1131

\section{Hintergrund und Fragestellung}

Mehr als zwei Drittel der Bettpartner von Schnarchern (mit und ohne Apnoen) beklagen eine schlechte Schlafqualität [1]. Neben der Verwendung von Ohrstöpseln kann die Behandlung des Schnarchers (z.B. mit nCPAP) eine Lösung dieses Problems sein. Wenngleich die CPAP-Therapie über vier Wochen die subjektive Schlafqualität des Bettpartners in einer Studie verbesserte, waren die objektiven Parameter der Schlafqualität unverändert [1].

Eine weitere Möglichkeit, die Störung der Nachtruhe zu unterbinden, besteht in der räumlichen Trennung des Bettpartners in der Nacht. Ziel der vorliegenden Untersuchung war die objektive Messung des Nachtschlafs der weiblichen Bettpartner ohne den Schnarcher.

\section{Methodik}

In die multizentrische Querschnittsstudie wurden 23 weibliche Personen ein- geschlossen, die über eine schlechte Schlafqualität aufgrund des chronischen Schnarchens ihrer Bettpartner klagten. Die Partnerschaften der Studienteilnehmer waren über mindestens ein Jahr stabil. Bei allen weiblichen Bettpartnern erfolgten innerhalb einer Woche zwei Polysomnografien in der häuslichen Umgebung, einmal mit und einmal ohne den schnarchenden Bettpartner.

\section{Ergebnisse}

30,4\% der Paare wurden ausgeschlossen, weil die Bettpartnerin selbst in mehr als 10\% der Schlafzeit schnarchte.

Bei Betrachtung der Schlafqualität der verbliebenen 16 Bettpartnerinnen zeigte sich lediglich ein geringer, signifikanter Unterschied in der Nacht ohne Bettpartner im Anteil des Non-REM-Schlaf-Stadiums 2 und im Aufwach-Index (Tab. 1). Während der gemeinsamen Nacht waren Erwachen und Arousals nicht zur Schnarchintensität des Bettpartners kor-

\begin{tabular}{|c|c|c|c|c|}
\hline \multicolumn{4}{|c|}{ Schlafqualität von Frauen mit und ohne schnarchenden Partner } & \multirow{2}{*}{$\begin{array}{c}\text { Tabelle } 1 \\
\text { p-Wert } \\
\text { (para- } \\
\text { metrisch) }\end{array}$} \\
\hline & $\begin{array}{l}\text { Nacht ohne } \\
\text { Partner }\end{array}$ & $\begin{array}{l}\text { Nacht mit } \\
\text { Partner }\end{array}$ & $\begin{array}{c}\text { p-Wert } \\
\text { (nicht para- } \\
\text { metrisch) }\end{array}$ & \\
\hline Schlafdauer insgesamt (min) & 359,8 & 332,5 & 0,147 & 0,355 \\
\hline Schlafleistung (\%) & 87,6 & 83,4 & 0,224 & 0,232 \\
\hline Latenz Stadium 3 (min) & 22,1 & 27,8 & 0,245 & 0,524 \\
\hline REM-Schlaf-Latenz (min) & 84,0 & 91,4 & 0,717 & 0,622 \\
\hline Non-REM-Schlaf Stadium 1 (\%) & 8,2 & 8,7 & 0,950 & 0,646 \\
\hline Non-REM-Schlaf Stadium 2 (\%) & 50,2 & 55,0 & 0,023 & 0,031 \\
\hline Non-REM-Schlaf Stadium 3-4 (\%) & 16,8 & 14,9 & 0,147 & 0,195 \\
\hline Non-REM-Schlaf Stadium 3-4 (min) & 59,1 & 50,5 & 0,217 & 0,235 \\
\hline REM-Schlaf (\%) & 24,8 & 21,4 & 0,196 & 0,179 \\
\hline Aufwach-Index & 4,0 & 4,8 & 0,046 & 0,086 \\
\hline Arousal-Index & 11,4 & 11,8 & 0,214 & 0,763 \\
\hline
\end{tabular}

reliert. Insgesamt fand sich kein klinisch relevanter Unterschied der objektiv gemessenen Schlafqualität während einer Nacht ohne den schnarchenden Bettpartner.

\section{Kommentar}

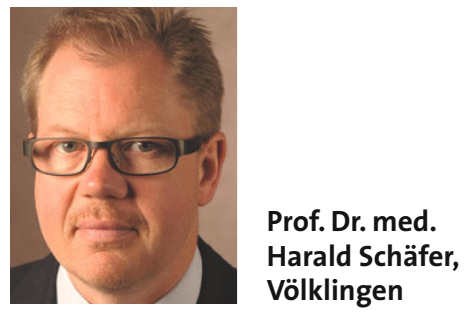

Bettpartner von Schnarchern bzw. unbehandelten Patienten mit Schlafapnoe beklagen sich häufig über eine subjektiv eingeschränkte Schlafqualität. Dies mag ein Grund dafür sein, warum die Initiative zur weiteren Diagnostik (und ggf. Therapieeinleitung) häufig vom Bettpartner ausgeht. Dass eine Behandlung mit nCPAP auch zur Verbesserung subjektiver und objektiver Schlafqualitätsparameter der Bettpartner führen kann, ist aus einigen Studien $[2,3]$ bekannt.

Dass in dieser Untersuchung eine Nacht ohne den schnarchenden Bettpartner keine relevante Auswirkung auf die Schlafqualität hatte, kann mehrere Ursachen haben. In erster Linie spielen sicherlich die Nacht-zuNacht-Variabilität und die letztlich sehr geringe Probandenzahl eine Rolle.

Die wichtigste Erkenntnis aus der vorliegenden Studie scheint jedoch zu sein, dass man zur Behandlung den (oder die) Richtigen auswählt. Bei immerhin einem Drittel der Paare scheint das Problem der nächtlichen Atmungsstörung beim vermeintlich nicht schnarchenden Bettpartner mindestens ebenso groß und möglicherweise für die eingeschränkte Schlafqualität verantwortlich zu sein.

\section{Literatur}

1. McArdle $\mathrm{N}$ et al. Partners of patients with sleep apnoea/hypopnoea syndrome: effect of CPAP treatment on sleep quality and quality of life. Thorax 2001; 56:513-518

2. Doherty LS et al. Impact of nasal continuous positive airway pressure therapy on the quality of life of bed partners of patients with obstructive sleep apnea syndrome. Chest 2003;124:2209-2214

3. Kiely JL et al. Bed partners' assessment of nasal continuous positive airway pressure therapy in obstructive sleep apnea. Chest 1997;111:12611265 